Review Article

\title{
A situation model of integrated health information platform in India: an anticipated review
}

\author{
Mogan $\mathrm{Ka}^{1 *}$, Aravind Gandhi Periyasamy ${ }^{2}$, Venkatesh U. ${ }^{1}$, Jugal Kishore $^{1}$
}

\begin{abstract}
${ }^{1}$ Department of Community Medicine, Vardhman Mahavir Medical College and Safdarjung Hospital, New Delhi, Delhi, India

${ }^{2}$ Department of Community Medicine and School of Public Health, Post Graduate Institute of Medical Education and Research, Chandigarh, India
\end{abstract}

Received: 02 January 2020

Revised: 10 February 2020

Accepted: 11 February 2020

\section{*Correspondence:}

Dr. Mogan Ka,

E-mail: moganka1994@gmail.com

Copyright: (C) the author(s), publisher and licensee Medip Academy. This is an open-access article distributed under the terms of the Creative Commons Attribution Non-Commercial License, which permits unrestricted non-commercial use, distribution, and reproduction in any medium, provided the original work is properly cited.

\begin{abstract}
Health system in India is fragmented with different kinds of health care providers engaged in the delivery of health services across rural and urban areas. The integration of health information in a single platform aids in strengthening the public health surveillance systems, better decision support system, improvement in the efficiency and effectiveness in the context of health education and monitoring. In order to integrate the public health data and strengthen the surveillance and monitoring, the integrated health information platform (IHIP) was launched as pilot project following the report of the joint monitoring mission of the World Health Organization. IHIP integrates the electronic health records of the citizens on a pan-India basis aids in providing a comprehensive health profile of the population. Integrated disease surveillance programme (IDSP) module of integrated health information platform (IHIP) was the earliest initiative of integrating various health programmes. It is a web-based real-time, village wise, case-based electronic health information system that will aim to provide details about epidemic-prone diseases with geographical information system (GIS) thus reducing the morbidity and mortality and lessening disease burden in the populations. GIS information obtained via IHIP provides information in the form of a geographical map as interface data with real-time monitoring thus contributes to Geospatial Epidemiology in addition to information by IDSP. This article aims to describe the anticipated model of IHIP in the context of providing 'One Health' by the integration of health sectors.
\end{abstract}

Keywords: GIS integration, Integrated disease surveillance programme, Integrated health information platform, One health, Real-time monitoring

\section{INTRODUCTION}

Health system in India is fragmented with different kinds of health care providers engaged in the delivery of health services across rural and urban areas. With increase in progress towards computerization in public health services, it is essential to integrate the data to gain meaningful information for planning health programmes.
The integration of health information in a single platform aids in enabling hospitals, physicians, laboratories, pharmacies, and other health services providers to deliver faster, more accurate, safer, higher quality and less redundant medical care to patients in the country. ${ }^{1}$ The current trend in collecting information via digital or by electronic conversion of health records helps in combining and correlating information in different health programmes. ${ }^{2}$ In 2016, India had tabled a resolution at 
World Health Organization for mobile health, which was supported by over 30 nations, following the report of Global Observatory for eHealth. It indicates the intent of India towards digital health. As a part of International Health Regulations (IHR) core capacity building and to strengthen public health surveillance systems, India needs a comprehensive information system to monitor emerging and re-emerging public health threats, disasters and mass events. It is essential to integrate sources of health information because public health surveillance is not only surveillance collection, analysis, interpretation but also the dissemination of health data for the planning, implementation and evaluation of public health action. ${ }^{3,4}$ Thus it is essential to get a state-of-the-art single operating picture of health profile by integrating all the health-related information. ${ }^{5}$

\section{WHAT IS INTEGRATED HEALTH INFORMATION PLATFORM?}

The Integrated Health Information Platform (IHIP) provides a comprehensive health profile of the population. It integrates the Electronic Health Records (EHRs) of the citizens on a pan-India basis enabling better continuity of care, secure and confidential health data/records management, better diagnosis of diseases, reduction in patient re-visits and even prevention of medical errors, better affordability, optimal information exchange to support better health outcome, better decision support system, and thus eventually facilitating improvement in the reforms of treatment and care of public health at National-Level. It functions by integrating communicable disease programmes such as National Vector Borne Disease Control Programme (NVBDCP), National Leprosy Eradication Program (NLEP), National AIDS Control Organisation (NACP), Revised National Tuberculosis Control Program (RNTCP), Anti-Microbial Resistance (AMR) surveillance, non-communicable disease programmes such as National Program for Prevention and Control of Cancer, Diabetes, CVD and Stroke (NPCDCS), National Tobacco Control Programme (NTCP), National Programme for Control of Blindness (NPCB), National Mental Health Programme (NMHP), Injury, trauma with National Animal Disease Reporting System (NADRS), Ministry of Environment, Forest and Climate Change (MoEFCC), Ministry of Agriculture and Farmers Welfare, Ministry of Earth Sciences and Ministry of Electronics and Information Technology. ${ }^{6}$

As a first step, the Integrated Disease Surveillance Programme (IDSP) module of the Integrated Health Information Platform (IHIP) was the earliest initiative of integrating various health programmes. It was soft launched in selected districts of 7 states as a pilot project by the Ministry of Health and Family Welfare (MoHFW), Government of India in 2018. It was launched to address the existing situation of 'electronic silos' in the health system and increase the efficiency of data exchange. The Integrated Health Information Platform (IHIP) in context to IDSP is a web-based real-time, village wise, casebased electronic health information system which will aim to provide details about epidemic-prone diseases with Geographical Information System (GIS) thus reducing the morbidity and mortality and lessening disease burden in the populations. ${ }^{7}$ IHIP aids in providing 'One Health' since around $60 \%$ of existing and $75 \%$ of emerging human infectious disease is zoonotic origin and a strong link between environment and Health. India is committed to provide 'One Health' as the One Health approach can be operationalized with effective collaboration and integration among various sectors within the complex health system. ${ }^{8}$ Hence IHIP is a prime initiative and a stepping stone for implementing a countrywide One Health Collaboration (OHC) policies and strategies.

Real-time monitoring with spatial configuration in terms of infectious disease is extremely important for initiating the investigation at the earliest. Geographical Information System (GIS) provides information in the form of a geographical map as interface data with real-time monitoring and providing geospatial epidemiology. ${ }^{9}$ Geospatial epidemiology provides the ability to describe and analyze geographic variations in diseases in the context of demographic, environmental, behavioral, socioeconomic, genetic, and infectious risk factors. It also helps to interpret geographic correlations of persons with their socioeconomic and demographic attributes and conduct public health surveillance. The real-time monitoring is vital in solving public health issues and has a wide impact globally in relation to acute infectious diseases such as Hepatitis A, water-borne diseases and even in non-communicable diseases. ${ }^{10}$

\section{HOW IT IS INTEGRATED WITH INTEGRATED DISEASE SURVEILLANCE PROGRAMME?}

Integrated Disease Surveillance Programme (IDSP) integrates, coordinates and correlates the information with respect to inter-sectoral and intra-sectoral organization, public and private, rural and urban health system. IDSP compiles the information in a single nodal point and facilitates sharing that information with other disease control programmes and stakeholders. However, the Center for Disease Control (CDC) and the Joint Monitoring Mission of the World Health Organization (WHO) reports IDSP have overabundance of fragmented datasets and to collect more epidemiological data for action. IHIP was implemented to bridge the gaps in IDSP by integrating the health information from the surveillance data of various disease control programmes including communicable and non-communicable diseases providing geospatial epidemiology. ${ }^{11}$ These aids in avoiding resource wastage, pooling of resources, providing quick information and response with better quality and efficiency in comparison to IDSP. Thus, IHIP integrates data obtained from both public and private health sectors providing signal for outbreak earlier than IDSP. IHIP provides the union health ministry, state health ministries, local governments, municipalities with 
real-time information on health surveillance from anywhere on any electronic device. IDSP captures aggregated paper-based data and provides a weekly surveillance report on specified diseases only. However, IHIP is designed to capture disaggregate data of 33 priority health conditions at various health care levels and links the data from S, P and L forms of IDSP. It provides a piece of comprehensive information with real-time monitoring of ongoing surveillance programmes. All data contained in IHIP has the public health surveillance attributes of person, place, time and the data are geocoded for geographic reference. The process of data reporting on IHIP under IDSP consists of the following two key phases. First step is verification of master data of health facilities (including examining of user IDs and passwords, creation of user profiles, health facility directory, etc.). This is a one-time initial activity to set up basic information. Second step is reporting disease surveillance data (including event alerts and outbreak) which is a continuous activity carried out in a real-time manner. ${ }^{11}$ For instance, hotspots of mosquito breeding sites provided by NVBDCP in specified area can be correlated with the vector borne disease outbreak, thus helps in planning intervention by integrating the information. Similarly, data collected by National Animal Disease Reporting System (NADRS) can be used in outbreak investigations of zoonotic diseases.

\section{CURRENT STATUS OF IHIP IN OUTBREAK?}

In addition to available resources of outbreak, IHIP provides Health condition alert that will be generated automatically depending upon the threshold set for each disease, automated epidemic curve plot and pathogen wise distribution of cases or when the number of laboratory confirmed cases for a particular disease cross threshold. Furthermore, hotspots can be identified from the clusters with the information obtained from GIS tagging of individual cases. In addition to outbreak detection, IHIP aids in fastening the outbreak investigation by selecting rapid response team from the IHIP portal via online, based on the availability of trained members in the particular outbreak area.

The complete information regarding the outbreak can be accessed by State and National levels on a real-time basis which helps in monitoring public health emergencies. ${ }^{6}$

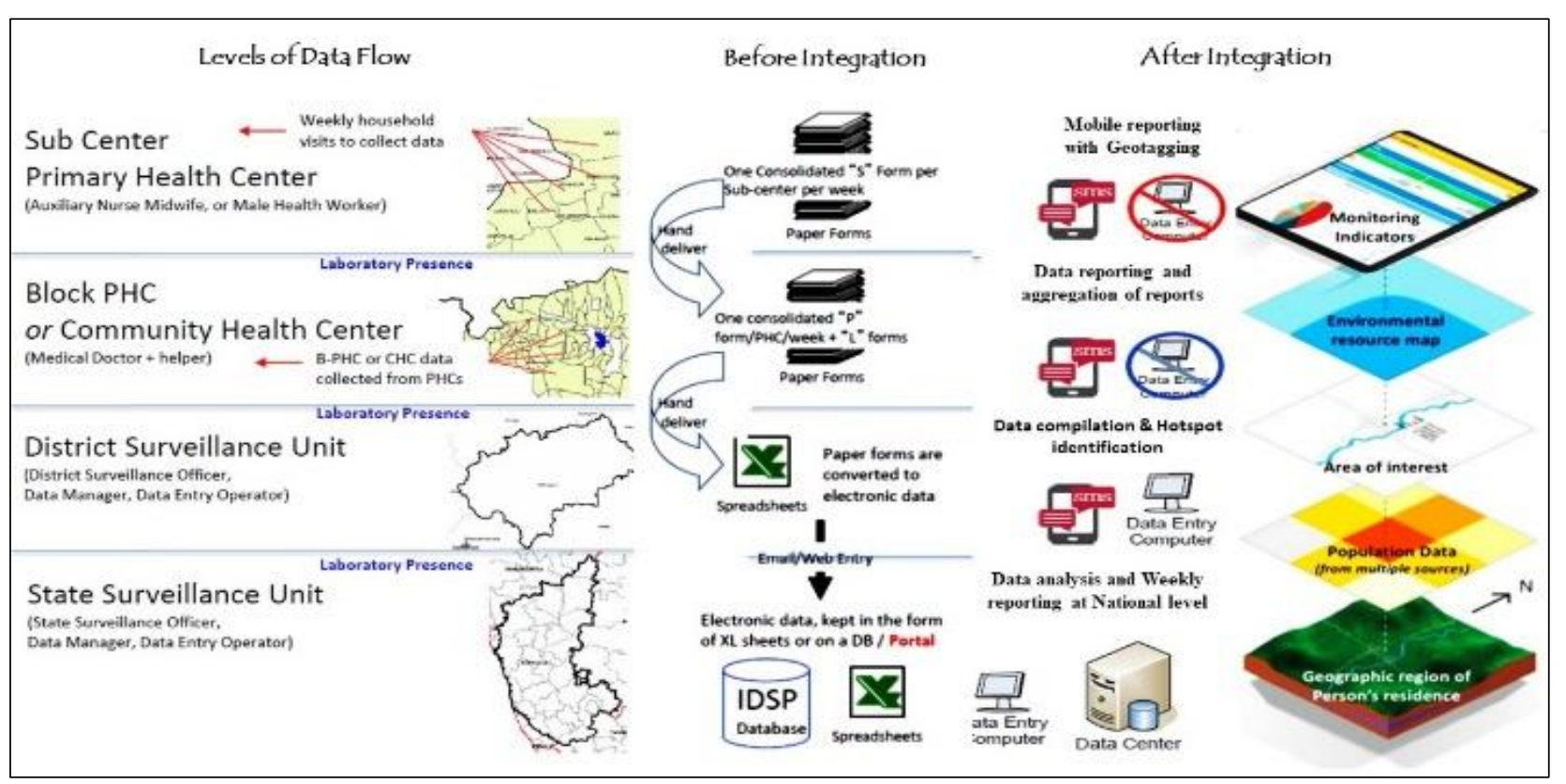

Figure 1: Conceptual framework of integration of IHIP with IDSP at various levels.

\section{INTEGRATION MODEL IN DEVELOPED NATIONS}

Researchers in developed countries like the United States, finds public health information system integration with Health Information Exchange (HIE) organizations that produces improvements in public health data leading to care coordination, preparedness, surveillance and workplace efficiency. In 2013, they found the investigation of electronic lab report messages finds data enriched by an HIE organization is more complete, compared to data from clinical systems. ${ }^{12,13}$ Web-Based
Integrated Public Healthcare Information System (PHIS) of Korea in 19 functional business areas with 47 detailed health programs found PHIS improved the efficiency and effectiveness in the context of health education and monitoring, clinical information management, administration and civil service, and system maintenance. $^{14}$

\section{CONCLUSION}

This paper has attempted to present the rationale of Integration of health sector information in context to IHIP 
and anticipated the outcome of IDSP model of IHIP. Thus, IHIP aids in providing 'One Health' and change the pace of data collection with real-time monitoring. IHIP would also provide an opportunity for health IT start-ups to host their innovative solutions for use by different stakeholders. However, the cost-effectiveness and the reliability of the data should be evaluated in the near future with the results from Phase 1.

Funding: No funding sources

Conflict of interest: None declared

Ethical approval: Not required

\section{REFERENCES}

1. EMR Standards Committee. Recommendations on electronic medical records standards in India. Available at: https://mohfw.gov.in/sites/default/files/ 24539108839988920051EHR\%20Standardsv5\%20Apr\%202013.pdf. Accessed 19 December 2019.

2. Janakiraman R, Park E, Demirezen E, Kumar S. The effects of health information exchange access on healthcare quality and efficiency: An empirical investigation. Mays Busin School Res Paper; 2017 Available at: https://ssrn.com/abstract=2915190. Accessed 19 December 2019

3. World Health Organization. Digital health. Draft resolution proposed by Algeria, Australia, Brazil, Estonia, Ethiopia, Germany, India, Indonesia, Israel, Italy, Luxembourg, Mauritius, Morocco, Panama, Philippines, and South Africa. 71st World Health Assembly agenda item. 2018:25;12:A71.

4. Nsubuga P, White ME, Thacker SB, Anderson MA, Blount SB, Broome CV, et al. Public health surveillance: a tool for targeting and monitoring interventions. Dis Control Priori Develop Coun. 2006;2:997-1018.

5. Balsari S, Fortenko A, Blaya JA, Gropper A, Jayaram M, Matthan R, et al. Reimagining Health Data Exchange: An application programming interface-enabled roadmap for India. J Med Inter Res. 2018;20(7):e10725.
6. Integrated Health Information Program (IHIP). Available at: https://www.nhp.gov.in/integrated health_information_program_mtl. Accessed 16 December 2019.

7. Guidelines of Soft launch Integrated Health Information Platform Integrated Disesase Surveillance Programme of Segment of November; 2018.

8. Yasobant S, Bruchhausen W, Saxena D, Falkenberg T. One health collaboration for a resilient health system in India: Learnings from global initiatives. One Health; 2019: 100096.

9. Fradelos EC, Papathanasiou IV, Mitsi D, Tsaras K, Kleisiaris CF, Kourkouta L. Health based geographic information systems (GIS) and their applications. Acta Inform Med. 2014;22(6):402.

10. Elliott P, Wartenberg D. Spatial epidemiology: current approaches and future challenges. Environ Health Pers. 2004;112(9):998-1006.

11. Suresh K. Integrated Diseases Surveillance Project (IDSP) through a consultant's lens. Ind J Pub Health. 2008;52(3):136-43.

12. Dixon BE, Siegel JA, Oemig TV, Grannis SJ. Electronic health information quality challenges and interventions to improve public health surveillance data and practice. Pub Health Rep. 2013;128(6):54653.

13. Kierkegaard P, Kaushal R, Vest JR. Applications of Health Information Exchange Information to Public Health Practice, AMIA Annu Symp Proc. 2014;2014:795-804.

14. Ryu S, Park M, Lee J, Kim SS, Han BS, Mo KC, et al. Web-based integrated public healthcare information system of Korea: development and performance. Healthcare Infor Res. 2013;19(4):314-23.

Cite this article as: Ka M, Periyasamy AG, Venkatesh U, Kishore J. A situation model of integrated health information platform in India: an anticipated review. Int J Community Med Public Health 2020;7:1197-200. 